\title{
Stage IIIB Ovarian Cancer AJCC v8
}

National Cancer Institute

\section{Source}

National Cancer Institute. Stage IIIB Ovarian Cancer A/CC v8. NCI Thesaurus. Code C139975.

Stage IIIB includes: T3b, N0/N1, M0. T3b: Ovarian cancer with macroscopic peritoneal metastasis beyond pelvis $2 \mathrm{~cm}$ or less in greatest dimension with or without metastasis to the retroperitoneal lymph nodes. N0: No regional lymph node metastasis. N1: Positive retroperitoneal lymph nodes only (histologically confirmed). M0: No distant metastasis. (AJCC 8th Ed.) 